\title{
Tolerance of 16-Channel Dense Wavelength Division Multiplexing System to Fibre Dispersion and Four-Wave Mixing under Varying Capacities and Channel Spacing
}

\author{
Oluwaseun Tooki, Robert Abolade, and David Aborisade
}

\begin{abstract}
An essential feature of a long-haul Optical Communication System (OCS) is to transmit huge volumes of data over long distances. In OCS, fiber dispersion and FourWave Mixing (FWM) play negative roles in achieving this, as the propagation of the light signal, associated with the pulse, travels at varying speeds. Dense Wavelength Division Multiplexing (DWDM) system is not spared from these identified limitations. In this work, a mathematical analysis for the dispersion and FWM was presented and validated through simulation software. The research was designed and implemented using an optical simulation software tool, OptiSystem 17.0. Transmission parameters of dispersion and Nonlinear (NL) effects due to refractive index, FWM, were considered. The research was carried out on a $125 \mathrm{~km}$-long fiber link and was investigated under a 16-channel DWDM system. The effects of identified limitations on the performance of the DWDM system with channel arrangement of 100, 50, and $25 \mathrm{GHz}$ were further explored. This work compared the DWDM system under these different channel arrangements. The transmission performance of the DWDM system was evaluated based on signal power, noise power, and eye pattern. The results obtained show that any increase in capacity causes significant decreases in the performance of the DWDM system. It was, however, noted that noise power is independent of an increase in bit rate. The results obtained further revealled that the launched power of the system must be reduced for better performance of the DWDM system and improved immunity against identified limitations.
\end{abstract}

Keywords - Dense Wavelength Division Multiplexing (DWDM), Fibre Dispersion, Four-Wave Mixing (FWM), Optical Communication System (OCS).

\section{INTRODUCTION}

Optical Communication System (OCS) is a system whereby data and information are moved in a form of light pulse through an optical fiber from one destination to another [1]. An OCS is the bedrock of todays' long-distance communication networks based on its high capacity and moderate loss in the transmission of signals over long distances [2]. Transmission of a signal via coaxial cable is fast being replaced with optical fiber due to its intrinsic

Submitted on December 14, 2021

Published on February 14, 2022.

O. O. Tooki, Air Force Institute of Technology, Nigeria.

(corresponding e-mail: o.tooki@afit.edu.ng)

R. O. Abolade, Ladoke Akinola University of Technology, Nigeria.

(e-mail: roabolade@lautech.edu.ng

D. O. Aborisade, Ladoke Akinola University of Technology, Nigeria

(e-mail: doaborisade76@lauech.edu.ng). advantages of long-distance signal transmission, exceptional bandwidth, and signal security. However, Nonlinear (NL) impairment of Four-Wave Mixing (FWM), in fibre degrades channel capacity and high data-rate transmission of the Dense Wavelength Division Multiplexing (DWDM) system [3]. This impairment sets a restriction fibre on the highest signal power that can be transmitted. [4]. These nonlinearities in the fiber can be classified into two, one due to scattering and the other due to refractive index [5]. SelfPhase Modulation, Cross-Phase Modulation, and FWM are nonlinearities caused by a change in the refractive index. Also, NL attributes that occur as a result of scattering include Stimulated Brillouin Scattering and Stimulated Raman Scattering [2]. FWM occurs when four waves propagate concurrently inside a fibre [5].

In OCS, signals transmitted over long-haul suffered from not only nonlinearity but also dispersion. Optical signal propagation in fibre is governed by dispersion and nonlinearity [6], [7]. Dispersion occurs when light pulses spread out while traveling through the fibre. This often leads to the broadening of the optical signal. Dispersion can either be modal, chromatic, or polarization. All the three dispersion types affect both Singlemode Fibre (SMF) and multimode fibre; except for SMF that is unaffected by modal dispersion. Dispersion distorts signals along with the link. To mitigate against these impairments, dispersion, and fibre nonlinearities, the design of OCS requires the need to adopt efficient dispersion management techniques.

This paper investigated the influence of dispersion and FWM on the performance of DWDM. DWDM is a capacity system used to multiplex different optical signals in one fiber for transmission over long distances. For the nonlinearities, special focus was given to FWM which is the most critical of them all. It affects both the transmission distance and capacity through long distances. In DWDM based system, the FWM effect increases with a decrease in channel spacing/arrangement [3].

Also, this paper investigated this limitation and proffer solutions through analytical modeling and simulation analysis. Mathematical analyses of dispersion and FWM were carved out. Also, studied is the response of the system to increase in capacity in the presence of dispersion and FWM.

Dispersion is one of the main parameters that cause an increase in pulse width broadening and consequently decrease transmission rates [8]. Optical frequency determines the medium response when an electromagnetic wave reacts with the electrons of a dielectric phenomenon 
called chromatic dispersion. On a fundamental level, the origin of chromatic dispersion is related to the characteristic resonant frequencies at which the medium absorbs the electromagnetic radiation through oscillations of bound electrons [9]. Three optical windows are currently in use in optical transmission for DWDM are $850 \mathrm{~nm}, 1310 \mathrm{~nm}$, and $1550 \mathrm{~nm}[10]$.

\section{RELATED WORKS}

Researchers such as [11] carried out performance analysis on ultralong-haul DWDM systems combining optical single-sideband modulation and orthogonal frequency division multiplexing to requite for chromatic dispersion. However, the researchers were silent about the effect of NL attributes on OCS. Reference [12] carried out a performance analysis of a 40 Gbps (GigaBits Per Second) DWDM system. The drawback of the proposed design is the complexity of the transmitter architecture. Reference [13] modeled a Digital Signal Processing NL impairment tolerant $100 \mathrm{Gbps}$ for long-distance optical network. The researchers were not specific about the type of impairment suitable with the developed model. The work of [14] proposed the use of a Differential Quadrature Phase Shift Keying (QPSK) modulation scheme to mitigate the impact of NL effects on the performance of DWDM system. A comparison was made with two variants of the DQPSK modulation scheme. However, the researchers are not specific about the NL attributes that the proposed method will suppress. Also, they were silent about the impact of dispersion.

The review of [15] discusses two methods, optical regeneration schemes for the compensation of nonlinearities effects and the use of dispersion management methods to enhance the scalability of the DWDM system. Although the review was extensive, the researchers failed to consider the choice of tighter channel spacing such as $25 \mathrm{GHz}$.

\section{METHODOLOGY}

The simulation results were solved by applying the Sellmeier equation for the dispersion and Schrodinger equations for the NL.

\section{A. Mathematical Analysis of Dispersion}

The Mathematical expression for dispersion was derived from the Sellmeier equation (7):

$$
n^{2}(\chi)=1+\sum_{j=1}^{k} \frac{B_{j} \chi_{j}^{2}}{\chi_{j}^{2}-\chi^{2}}
$$

where $n(\chi)=$ Frequency Dependence of the Refractive index, $\chi_{j}=$ Resonance Frequency, $B_{j}=$ Strength of $j$ th resonance.

By increasing the mode-propagation constant $\beta$ the effect of fibre dispersion was realized using the Taylor series to calculate the pulse spectrum's frequency $f_{0}$ is given as:

$$
\beta(\chi)=n(\chi) \frac{\chi}{c}=\beta_{0}+\beta_{1}\left(\chi-2 \pi f_{0}\right)+\frac{1}{2} \beta_{2}\left(\chi-2 \pi f_{0}\right)+\ldots .
$$

The relationship between the refractive index $n(\chi)$ with the parameters $\beta_{1}$ and $\beta_{2}$ are expressed as:

$$
\begin{gathered}
\beta_{1}=\frac{1}{v_{g}}=\frac{n_{g}}{c}=\frac{1}{c}\left(n+\chi \frac{d n}{d \chi}\right) \\
\beta_{2}=\frac{1}{c}\left(2 \frac{d n}{d \chi}+\chi \frac{d^{2} n}{d \chi^{2}}\right)
\end{gathered}
$$

where $v_{g}=$ group velocity, $n_{g}=$ group index. Note that $v_{g}$ varies with wavelength. The group velocity $v_{g}$ can be obtained by substituting $\beta_{1}$ in (3). The $\beta_{2}$ parameters, represent group velocity which induced the pulse broadening. The occurrence refers to as the group dispersion velocity dispersion parameter. The other dispersion parameter considered is represented by $\mathrm{D}$. The relationship between it and $\beta_{2}$; and is expressed as:

$$
D=\frac{d \beta_{2}}{d \lambda}=-\frac{2 \pi c}{\lambda^{2}} \beta_{2}=-\frac{\lambda}{c} \frac{d^{2} n}{d \lambda^{2}}
$$

\section{B. Mathematical Analysis of FWM Effect}

The FWM was implemented by solving Schrodinger equations with a special focus on the most severe. From Maxwell Wave Equation:

$$
\nabla^{2} E-\frac{1}{c^{2}} \frac{\partial^{2} E}{\partial t^{2}}=\frac{1}{\varepsilon_{0} C^{2}} \frac{\partial^{2} P}{\partial t^{2}}
$$

where $\varepsilon_{0}$ is the permittivity of free space, and the speed of light is denoted by $\mathrm{C}$.

The sum of Linear Polarization $\left(\mathrm{P}_{\mathrm{L}}\right)$ and NL Polarization $\left(\mathrm{P}_{\mathrm{NL}}\right)$ constitute the total Polarization, $\mathrm{P}$.

$$
P=P_{L}+P_{N L}
$$

FWM is NL polarized and better described from the third-order polarization [2]:

$$
P_{N L}=\varepsilon_{0} X^{(3)} \vdots E E E
$$

$\mathrm{P}_{\mathrm{NL}}$ is the induced NL polarization while $\mathrm{E}$ is the electric field.

In general, FWM is polarization-dependent, and a whole vector must be developed. The NL parameter, $\gamma$, can be expressed as:

$$
\gamma=\frac{2 \pi}{\lambda}\left(\frac{n_{2}}{A_{e f f}}\right) W^{-1} k^{-1}
$$


where $A_{\text {eff }}$ is the effective cross-section area of the fibers, $n_{2}$ is the NL refractive index for silica fibers and $\lambda$ is the wavelength of light.

To understand the FWM effect, the DWDM signal is considered to be a sum of the nonlinear polarization, $\mathrm{P}_{\mathrm{NL}}$, which is expressed as:

$$
P_{N L}=\varepsilon_{0} X^{(3)} E^{3}
$$

and the signal of an electric field can be written as:

$$
E=\sum_{p=1}^{n} E_{p} \cos \left(\omega_{p} t-k_{p} z\right)
$$

substituting (10) into (11):

$$
\begin{gathered}
P_{N L}=\varepsilon_{0} X^{(3)} \sum_{a=1 b=1 c=1}^{n} \sum^{n} \sum_{a}^{n} E_{a} \cos \left(w_{a} t-k_{a} z\right) \\
\cdot E_{b} \cos \left(w_{b} t-k_{b} z\right) E_{c} \cos \left(w_{c} t-k_{c} z\right)
\end{gathered}
$$

The number and the optical power of the newly generated signals are given as:

$$
G=\frac{N^{2}}{2}(N-1)
$$

where $\mathrm{N}$ is the number of channels and $\mathrm{G}$ is the number of newly generated sidebands. For the 16-channel DWDM system considered, G equals 1920.

\section{Simulation Setup}

The investigation was carried out using the OptiSytem 17.0 simulator which closely realizes a fiber optic transmission system. The system's parameters used in the simulation are presented in Table I. The photodiode at the receiver generates a time-domain signal proportional to the optical power. To demonstrate the noise and distortion caused by optical amplifiers and fiber nonlinearity, photodiodes with a responsivity of $1 \mathrm{~A} / \mathrm{W}$ were considered. The signal was modulated using a Mach-zander modulator. and conversion of electrical signal into an optical signal for the optical channel. The multiplexer unit at the transmitter unit and the Demultiplexer at the receiving end acted as the optical combiner and optical splitter, respectively. The experiment was conducted on a $125 \mathrm{~km}$ fiber link designed with Erbium-Doped Fiber Amplifiers (EDFAs). The $125 \mathrm{~km}$ long fibre was made of a $100 \mathrm{~km}$ and $25 \mathrm{~km}$ Dispersion Compensation Fibre (DCF). The choice of a $125 \mathrm{~km}$ transmission link is that the effects of dispersion an FWM can be noticed at this distance. The DCF was used after the SMF to combat the accumulated dispersion over the link.

The attenuation constant used in the design is 0.25 and $5 \mathrm{~dB} / \mathrm{km}$ for the SMF and DCF fibre, respectively. The EDFA was introduced along with the $125 \mathrm{~km}$ link. The experiment was demonstrated on a 16-channel DWDM system. According to the broad absorption minimum, the third window is best suited for DWDM design. The experiment was built around the third transmission window of $1550 \mathrm{~nm}$ owning to its low attenuation characteristics. The channel spacing of the DWDM system for $100 \mathrm{GHz}$ starting from 193.1 to $194.6 \mathrm{THz}$ was considered. The effect of the impairments for a tighter channel with 50 and $25 \mathrm{GHz}$ was probed. The eye diagram, one of the performance metrics used, was used to analyse the overall quality of the communication link. The receiver has PIN diodes, which were employed to convert the optical signal back to an electrical signal. An electrical power meter visualizer was used to measure the signal and noise power. The oscilloscope visualizer displays the waveform of the signal level at different capacities starting from $10 \mathrm{Gbps}$ to $100 \mathrm{Gbps}$ at an interval of $10 \mathrm{Gbps}$.

\begin{tabular}{ccc} 
& \multicolumn{2}{c}{ TABLE I: OPTICAL TRANSMISSION PARAMETERS } \\
\hline S/N & Parameters & System Values \\
\hline 1 & Input Power & $10 \mathrm{dBm}$ \\
2 & Fibre Attenuation Constant & $0.25 \mathrm{~dB} / \mathrm{km}$ \\
3 & Fiber Dispersion Coefficient & $16.5 \mathrm{ps} / \mathrm{nm} / \mathrm{km}$ \\
4 & Effective fibre Core Area & $70 \mu \mathrm{m}$ for SMF \\
5 & Reference Wavelength & $1550 \mathrm{~nm}$ \\
6 & Transmission Distance & $125 \mathrm{~km}$ \\
7 & Numbers of Channels & 16 \\
8 & EDFA gain & $20 \mathrm{~dB} / 100 \mathrm{~km}$ for SMF \\
9 & EDFA Noise Figure & $15 \mathrm{~dB} / 25 \mathrm{~km} \mathrm{for} \mathrm{DCF}$ \\
10 & Channel Spacing & $6 \mathrm{~dB}$ \\
11 & Capacity & $100 \mathrm{GHz}$ \\
12 & Receiver Responsivity & $10,20,30, \ldots, 100 \mathrm{Gbps}$ \\
\hline
\end{tabular}

\section{RESULTS AND DISCUSSION}

This paper investigated the tolerance of a 16-channel DWDM system to dispersion and fibre nonlinearity of FWM. Table II shows the signal and noise power obtained over different capacities ranging from 10 to 100 Gbps at an interval of $100 \mathrm{Gbps}$. The graphical representations of the signal amplitude and the corresponding Noise amplitude for 10, 50, and $100 \mathrm{Gbps}$ capacities are as shown in Fig. 1-3 respectively. For $100 \mathrm{GHz}$ channel spacing/arrangement, the signal power reduces with an increase in capacity with an exception to $70 \mathrm{Gbps}$ DWDM system. The signal power levels obtained for $100 \mathrm{GHz}$ spacing, at different capacities, starting from $10 \mathrm{Gbps}$ to $100 \mathrm{Gbps}$, at an interval of $10 \mathrm{Gbps}$ are $12.60,12.98,14.65,15.84,16.71,17.85,16.14$, $18.34,19.57$, and $20.81 \mathrm{dBm}$, respectively. This is shown in Table II. The obtained results depict that the system becomes susceptible to both dispersion and FWM with an increase in capacity. In addition, the FWM effect is also of concern on the account of its low signal power threshold, and it deteriorates the system's performance with an increase in the number of channels. Noise power results obtained over the same range fell within the value of -76.25 $\pm 2 \mathrm{dBm}$ for $100 \mathrm{GHz}$ channel spacing. Tables III and IV show the results of signal and noise power for the 50 and 25 $\mathrm{GHz}$ channel spacing at different capacities. The noise signal obtained under 50 and $25 \mathrm{GHz}$ channel spacing is in the range of $-67.04 \pm 2 \mathrm{dBm}$ and $-67.42 \pm 2 \mathrm{dBm}$, respectively. This further reinstates the observation that the noise power is independent of noise power to increase in capacity.

The eye diagrams obtained from the 16-Channel DWDM system for the input signals of 25, 20, 10, 5, and $3 \mathrm{dBm}$ are as shown in Fig. 4 to 8 . These diagrams determine the tolerance of the 16-DWDM system to signal impairment. 
The eye patterns in Fig. 4 and 5 showed how distorted the eye-opening and eye margins of the signals.

This was linked to the effect of dispersion and FWM. Fig. 6 shows the system performance at a reference input signal of $10 \mathrm{dBm}$. The dispersion and the FWM are responsible for the reduction in performance. However, when the input signal was reduced to 5 and $3 \mathrm{dBm}$ as shown in Fig. 7 and 8 ; the eye diagram is clearer when compared with the system transmitting above launch power greater than 10 $\mathrm{dBm}$.

TABLE II: SignAl AND NOISE POWER AT DiFFERENT CAPACITIES FOR $100 \mathrm{GHz}$ CHANNEL SPACING

\begin{tabular}{ccccccccccccc}
\hline \hline CAPACITY (GBPS) & 10 & 20 & 30 & 40 & 50 & 60 & 70 & 80 \\
\hline SigNAL POWER (DBM) & -12.6 & -12.94 & -14.65 & -15.84 & -16.71 & -17.85 & -16.14 & -18.34 & -19.57 & -20.81 \\
NOISE POWER (DBM) & -76.15 & -75.59 & -75.41 & -77.76 & -76.47 & -77.73 & -78.69 & -74.47 & -76.25 & -76.74 \\
\hline \hline
\end{tabular}

TABLE III: SignAL AND NOISE POWER AT DifFERENT CAPACITIES FOR 50 GHz CHANNEL SPACING

\begin{tabular}{ccccccccccccc}
\hline \hline CAPACITY (GBPS) & 10 & 20 & 30 & 40 & 50 & 60 & 70 & 80 & 90 \\
\hline SignAL POWER (DBM) & -3.79 & -3 & -3.49 & -3.67 & -2.79 & -2.49 & -2.81 & -4.8 & -4.75 & -5.2 \\
NOISE POWER (DBM) & -67.87 & -67.24 & -67.52 & -67.04 & -69.46 & -68.14 & -67.3 & -65.38 & -67.01 & -67.14 \\
\hline \hline
\end{tabular}

TABLE IV: SignAL AND NOISE POWER AT DIFFERENT CAPACITIES FOR 25 GHz CHANNEL SPACING

\begin{tabular}{cccccccccccccc}
\hline \hline CAPACITY (GBPS) & 10 & 20 & 30 & 40 & 50 & 60 & 70 & 80 & 90 & 100 \\
\hline SIGNAL POWER (DBM) & 4.84 & 2.34 & 3.57 & 0.66 & 1.52 & 1.87 & 3.23 & 2.5 & 1.22 & 1.5 \\
NOISE POWER (DBM) & -66.87 & -69.14 & -68.88 & -66.6 & -67.8 & -70.02 & -65.86 & -70.31 & -67.42 & -66.05 \\
\hline \hline
\end{tabular}

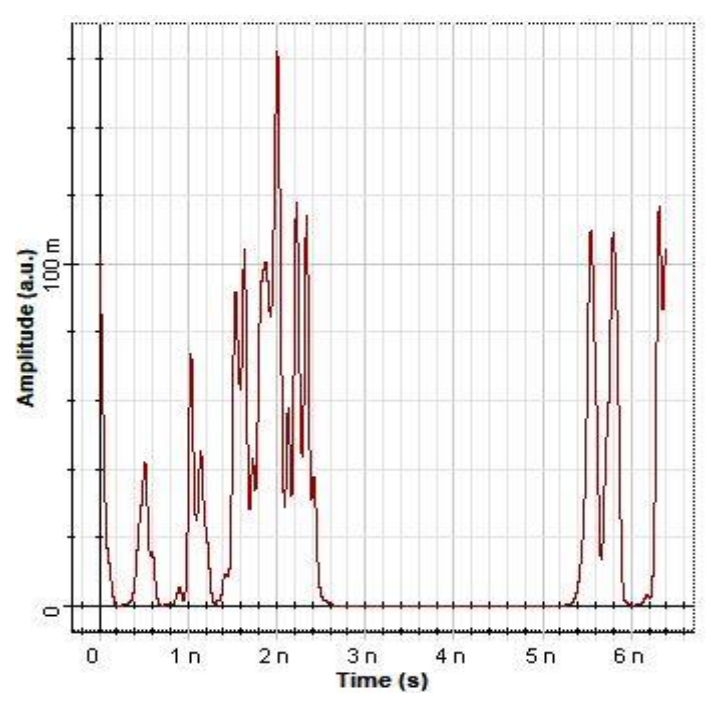

(a)

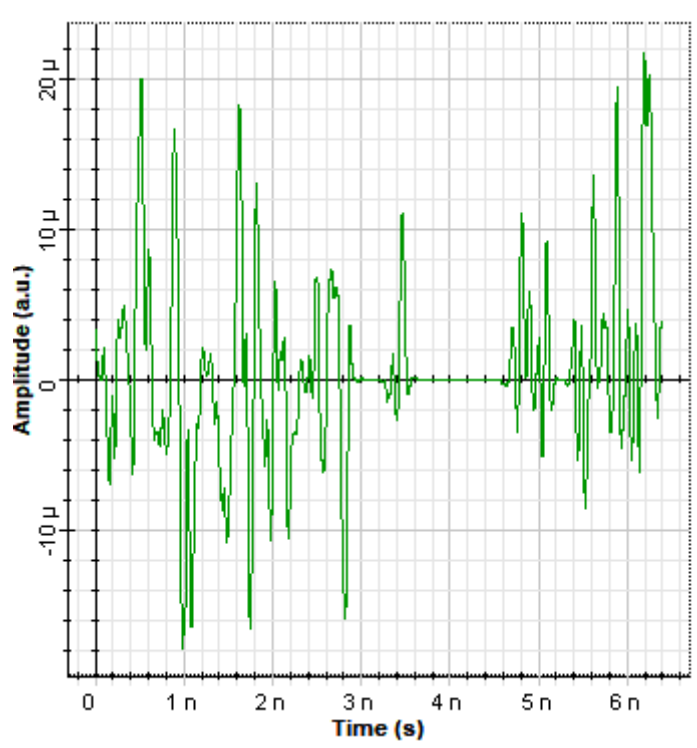

(b)

Fig. 1. Signal Amplitude (a) and Noise Amplitude (b) for $10 \mathrm{Gbps}$ under $100 \mathrm{GHz}$ Channel Arrangement.

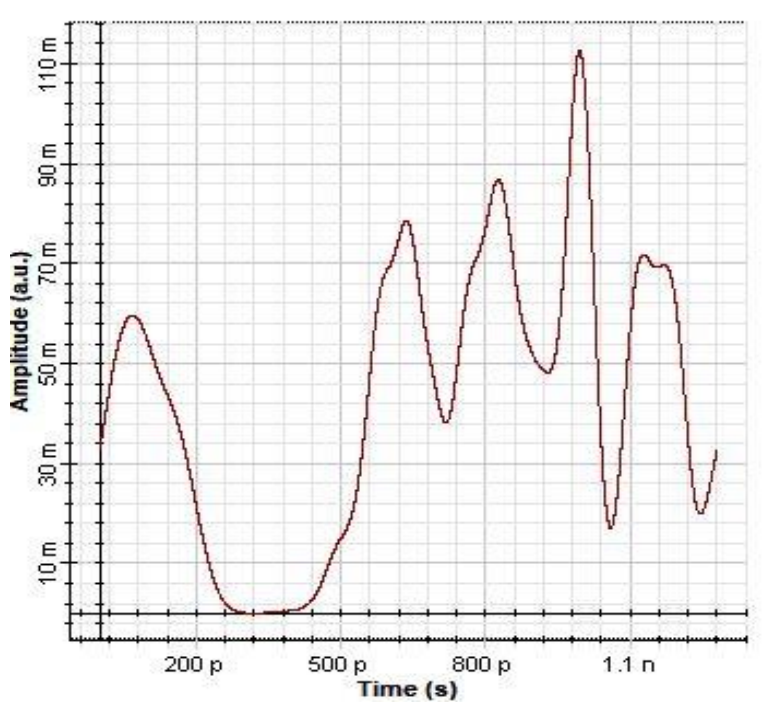

(a)

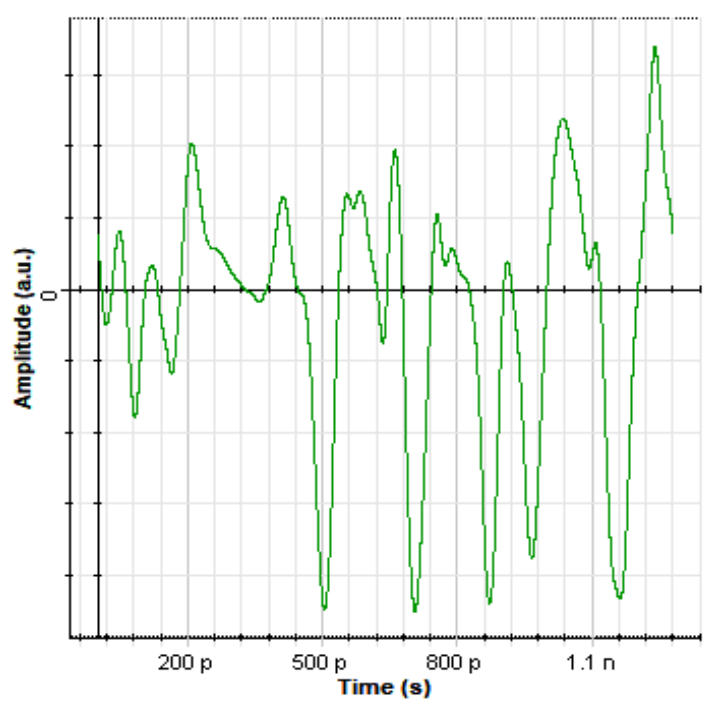

(b)

Fig. 2. Signal Amplitude (a) and Noise Amplitude (b)for 50 Gbps under $100 \mathrm{GHz}$ Channel Arrangement. 


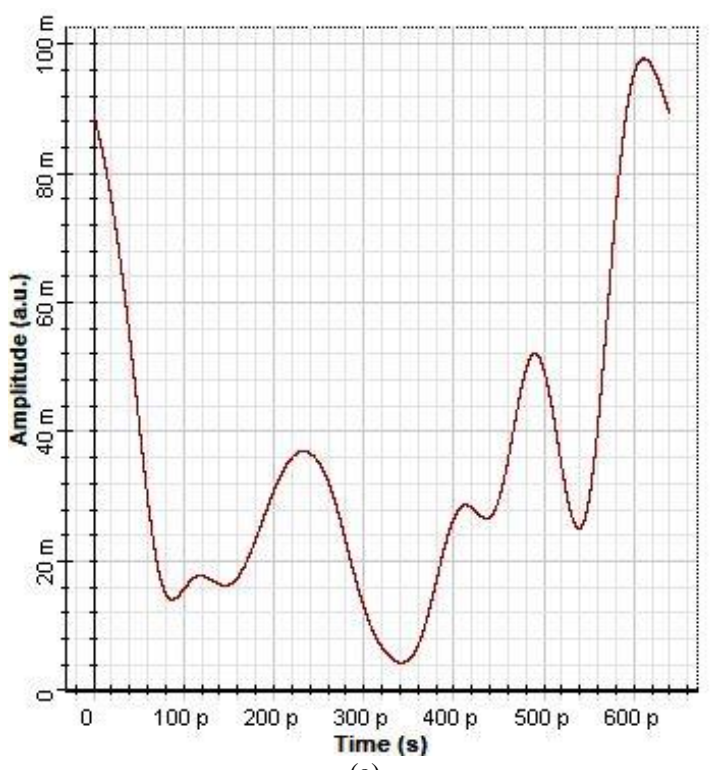

Fig. 3. Signal Amplitude (a0 and Noise Amplitude(b) for $100 \mathrm{Gbps}$ under $100 \mathrm{GHz}$ Channel Arrangement.

(b)

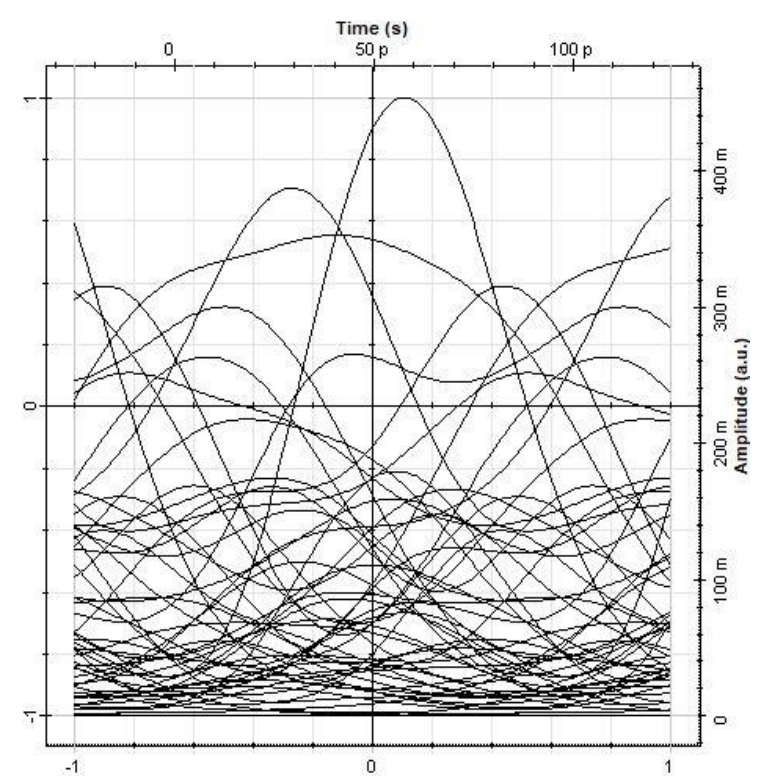

Fig. 4. Eye Diagram of Received Signals at Launch Power of $25 \mathrm{dBm}$.

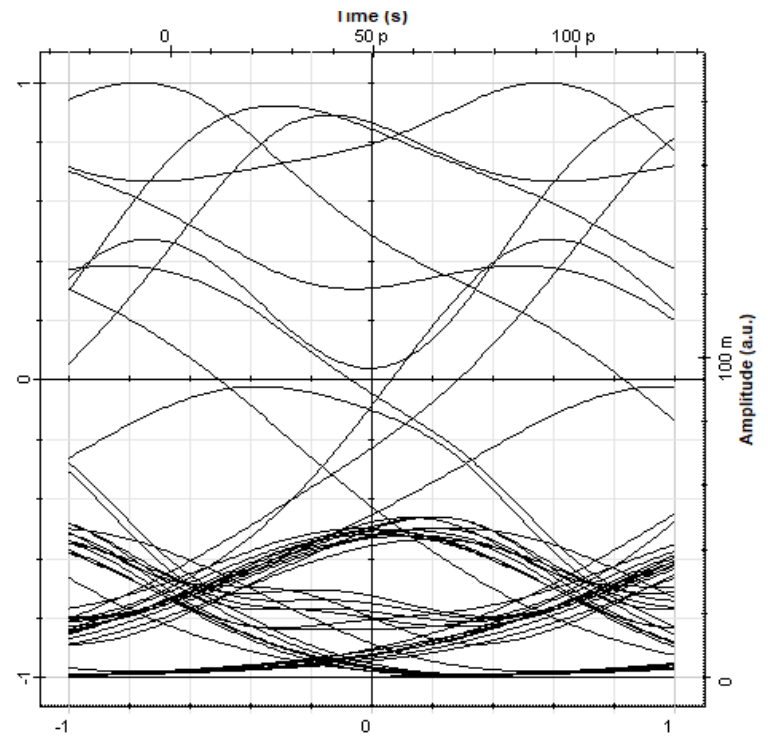

Fig. 6. Eye Diagram of Received Signals at Launch Power of $20 \mathrm{dBm}$.
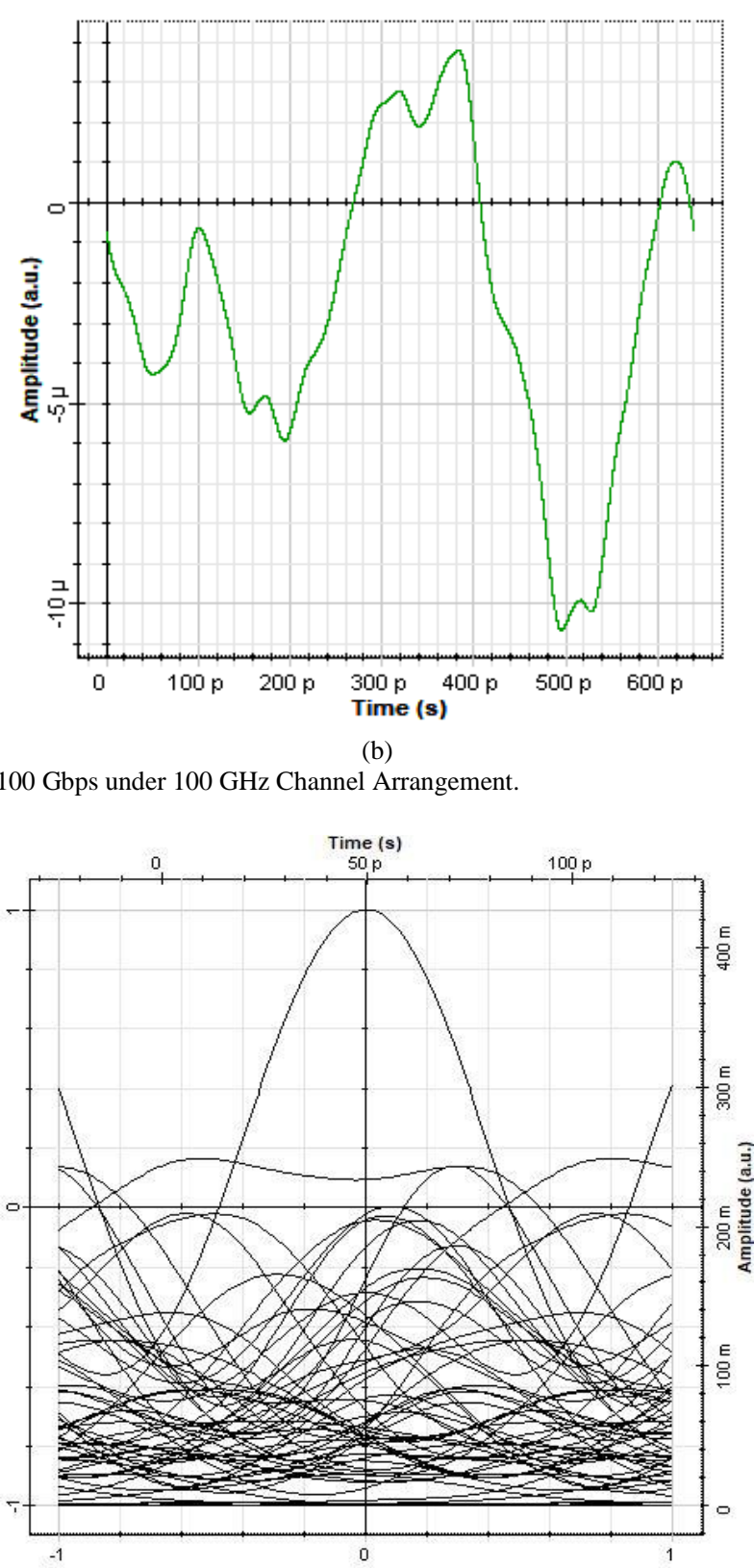

Fig. 5. Eye Diagram of Received Signals at Launch Power of $20 \mathrm{dBm}$.

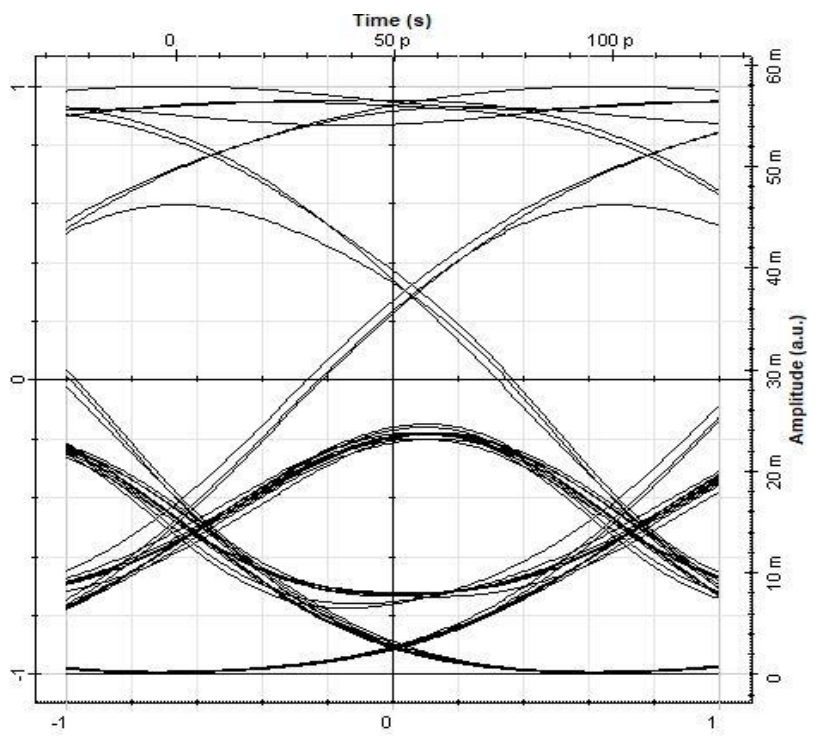

Fig. 7. Eye Diagram of Received Signals at Launch Power of $5 \mathrm{dBm}$. 


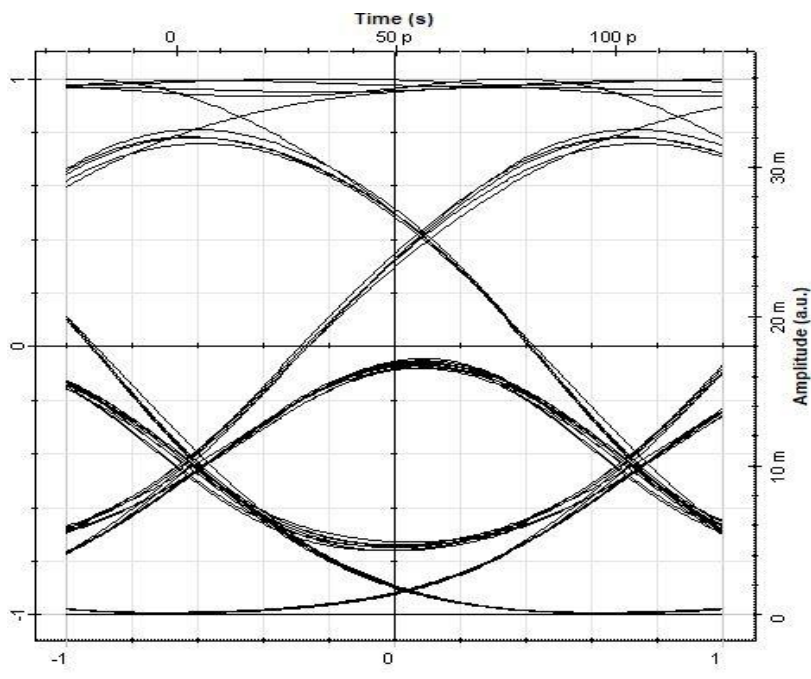

Fig. 8. Eye Diagram of Received Signals at Launch Power of $3 \mathrm{dBm}$.

\section{CONCLUSION}

In this paper, the impact of dispersion and FWM on the performance of the 16-Channel DWDM system transmitting over $125 \mathrm{~km}$ was investigated. The design has been demonstrated and the impact of dispersion and FWM on the performance of DWDM operating with 100, 50, and 25 $\mathrm{GHz}$ channel arrangement for varying capacities. The signal power increased with an increase in the capacity of the system. However, it was confirmed that the noise power is independent of any increase. In addition, it was observed that as the capacity increased, the performance of the system decreases. This was linked to the dispersion and the FWM effect. The effects of these identified impairments can be compensated for by reducing the launch power simultaneously with an increase in capacity. The proposed technique is best suited for DWDM systems with channel spacing of 100 and $50 \mathrm{GHz}$ channels spacing. It was noticed that the system with $25 \mathrm{GHz}$ spacing shows a considerable high FWM effect. It is recommended that $3 \mathrm{dBm}$ lunched power for a 16-channel DWDM system transmitting over a long haul of $125 \mathrm{~km}$ could be used to suppress negative attributes of FWM and dispersion thereby enhancing the performance of the system.

\section{CONFLICT OF INTEREST}

Authors declare that they do not have any conflict of interest.

\section{REFERENCES}

[1] Hiroshama N, Urvashi J, Vivekanand M. Performance investigation of kerr effects on to wdm fiber optical networks. IEEE International Conference on Recent Trends in Electronics Information Communication Technology, India May 20-21, 2017, pp. 2018-2022.

[2] Abolade RO, Tooki OO, and Aborisade DO. Review article on the mitigation of four-wave mixing in optical communication system, FUTA Journal of Engineering and Engineering Technology, April 2021;15(1):53-65. doi:10.51459/futajeet.2021.15.1.266.

[3] Ali F, Fazal M, Habib U, Khan Y, and Usman M. Modeling and minimization of fwm effects in dwdm-based long-haul optical communication systems. Photonic Network
Communications. Springer, September 2020;13:1-11. doi: 10.1007/s11107-020-00913-9.

[4] Satoshi Y, Yujia S, Zhixin L, Kyle RH, Bottrill FP, David J $\mathrm{R}$, and Periklis P. Mitigation of nonlinear effects on wdm qam signals enabled by optical phase conjugation with efficient bandwidth utilization. IEEE: Journal of Lightwave Technology, June 2016;17:1-8. doi: 10.1109/JLT.2016.2623740.

[5] Ali F, Khan Y, Qureshi SS. Transmission performance comparison of $16 * 100 \mathrm{Gbps}$ dense wavelength division multiplexed long haul optical networks at different advanced modulation formats under the influence of nonlinear impairments. Journal of Optical Communication, December 2018;10(2):165-172. doi:10.1515/joc-2018-0185.

[6] Jameel A, Hussaina A, Adeela F, Siyal MY, and Chongxiu Y. Optical signal processing using four wave mixing in highly nonlinear silicon nano-wire, Optik, October 2012;124: 34393442. doi:10.1016/j.ijleo.2012.10.030 Available: www.elsevier.de/ijleo.

[7] Dagar A, Dixit A, Kumar A and Kaur. Dispersion compensation in a 64-channel dense wavelength division multiplexing network. IEEE Xplore: Proceedings of the $4^{\text {th }}$ International Conference on Intelligent Computing and Control Systems 13-15 May 2020. doi: 10.1109/ICICCS48265.2020.9121140.

[8] Amiri I, Yupapin P, and Ahmed NZR. Mathematical model analysis of dispersion and loss in photonic crystal fibers. Journal of Optical Communication, March 2019;12:1-6. doi: 10.1515/joc-2019-0052.

[9] Agrawal GP. Nonlinear Fiber Optics, 4th ed. Academic Press, San Diego, USA, 2007, ch. 1, pp. 11-33.

[10] Keiser G, Optical fibre communications 2 nd ed. McGraw-Hill Inc. Publisher Indian 1991 ch. 10, pp. 321-339.

[11] Lowery AJ, Du LB, and Armstrong J. Performance of optical ofdm in ultralong-haul WDM lightwave systems. IEEE: Journal of Lightwave Technology, January 2007;25(1):131138.

[12] Sharan L, Shanbhag AG, and Chaubey VK. Design and simulation of modified duobinary modulated $40 \mathrm{Gbps} 32$ channel DWDM optical link for improved nonlinear performance. Cogent Engineering Journal, November 2016;3:1-12.doi:10.1080/23311916.2016.1256562.

[13] Irfan M, Ali F, Muhammad F, Habib U, Alwadie AS, Glowacz A, et al. DSP-Assisted Nonlinear Impairments Tolerant 100 Gbps Optical Backhaul Network for Long-Haul Transmission. Entropy MDPI pp. 1-15 September 2020. doi:10.3390/e22091062.

[14] Huszaník T, Turán J, and Ovseník L. On the carrier suppressed optical modulation methods for high capacity dwdm systems. IEEE 15th International Scientific Conference on Informatics, November 20-22, 2019 Poprad, Slovakia. doi: 10.1109/Informatics47936.2019.911925.

[15] Pallavi GP, Guan P, Li L, Lakoba TI, et al. Recent progress on optical regeneration of wavelength-division-multiplexed Data. IEEE Journal of Selected Topics in Quantum Electronics, March/April 2021;27(2). 